\title{
miR-29b promotes the osteogenic differentiation of mesenchymal stem cells derived from human adipose tissue via the PTEN/AKT/ $\beta$-catenin signaling pathway
}

\author{
TIAN XIA ${ }^{1,2}$, SHUANGHAI DONG ${ }^{1}$ and JIWEI TIAN ${ }^{2}$ \\ ${ }^{1}$ Shanghai General Hospital of Nanjing Medical University, Shanghai 200080; ${ }^{2}$ Department of Orthopedics, \\ Shanghai Jiahui International Hospital, Shanghai 200233, P.R. China
}

Received October 11, 2019; Accepted March 10, 2020

DOI: $10.3892 /$ ijmm.2020.4615

\begin{abstract}
Accumulating evidence has documented that microRNAs (miRNAs or miRs) function as important post-transcriptional regulators of the differentiation of mesenchymal stem cells (MSCs), including human adipose-derived mesenchymal stem cells (hADSCs); however, their roles in hADSC osteogenic differentiation require further investigation. The present study aimed to investigate the role of miRNAs in the osteogenic differentiation of hADSCs and to elucidate the underlying molecular mechanisms. Using an miRNA microarray, it was found that $24 \mathrm{miRNAs}$ were upregulated and 14 miRNAs were downregulated compared with the undifferentiated cells, and miR-29b-3p (miR-29b) was selected for further experiments. Functional experiments revealed that the upregulation of miR-29b by agomir-29b significantly enhanced alkaline phosphatase (ALP) activity and the mineralization of extracellular matrix (ECM), and led to an increase in the mRNA and protein levels of osteogenic marker genes, including runt-related transcription factor 2 (Runx2), osteopontin (OPN), osteocalcin (OCN) and bone sialoprotein (BSP), whereas the knockdown of miR-29b suppressed these processes. In addition, phosphatase and tensin homolog (PTEN), a negative regulator of the AKT/ $\beta$-catenin pathway, was identified as a direct target of miR-29b in the hADSCs. Moreover, it was observed that the overexpression of miR-29b activated the AKT/ $\beta$-catenin signaling pathway by inhibiting PTEN expression in the hADSCs. Most importantly, it was also found that the overexpression of PTEN reversed the promoting effects of miR-29b on osteogenic differentiation. On the whole, these findings suggest that miR-29b promotes the osteogenic differentiation of hADSCs by modulating the PTEN/AKT/ $\beta$-catenin signaling pathway. Thus, this miRNA
\end{abstract}

Correspondence to: Dr Jiwei Tian, Department of Orthopedics, Shanghai Jiahui International Hospital, 689 Guiping Road, Xuhui, Shanghai 200233, P.R. China

E-mail: jiweitianjw@163.com

Key words: microRNA-29b, differentiation, human adipose-derived mesenchymal stem cells, phosphatase and tensin homolog/ AKT/ $\beta$-catenin signaling pathway may be a promising target for the active modulation of hADSC-derived osteogenesis.

\section{Introduction}

Human adipose-derived mesenchymal stem cells (hADSCs), a newly recognized subpopulation of mesenchymal stem cells (MSCs), have the ability to differentiate into bone, cartilage, muscle and adipose tissue $(1,2)$, and have thus been suggested for use as potential seed cells clinically $(3,4)$. Osteogenic differentiation, a key function of hADSCs, plays a fundamental role in bone formation and remodeling. The elucidation of the molecular mechanisms of hADSCs osteogenic differentiation could promote the development of regenerative therapies for benign and malignant bone diseases. Several regulatory pathways, including the PI3K/AKT $(5,6)$, Wnt signaling (7) and transforming growth factor- $\beta$ (TGF- $\beta$ )/bone morphogenic protein (BMP) signaling pathways (8) have been implicated in the regulation of osteoblastic differentiation; however, the regulatory effects of the molecular pathways has yet to be investigated.

MicroRNAs (miRNAs or miRs) are a family of short, small, non-coding RNAs (an average size of 22 nucleotides), which negatively regulate target gene expression through either translation repression or RNA degradation $(9,10)$. Increasing evidence suggests that the osteoblastic differentiation of hADSCs is widely regulated by temporarily-expressed miRNAs. For instance, Luzi et al demonstrated that miR-26a inhibited the osteogenesis of hADSCs by suppressing the translation of SMAD1, a well-known osteogenic marker (11). Li et al demonstrated that miR-154-5p regulated the osteogenic differentiation of hADSCs under tensile stress by targeting Wnt11 (12). Moreover, the overexpression of miR-196a targeting HOXC8 has been shown to enhance osteogenic differentiation through the downregulation of hADSC proliferation (13). Despite these studies demonstrating the function of miRNA in the osteogenic differentiation of hADSCs, a limited number of studies have investigated the mechanisms of action of miRNAs during the osteogenic differentiation of hADSCs $(14,15)$.

In the present study, the differential expression of miRNAs was investigated using miRNA microarray analysis during the osteogenic differentiation of hADSCs. Moreover, the 
biological functions and mechanisms of action of miR-29b in the osteogenic differentiation of hADSCs were examined. The findings presented herein provide a better understanding of the molecular mechanisms underlying the complex processes of hADSCs in osteoblastic differentiation.

\section{Materials and methods}

Cells and cell culture. Adipose tissues were obtained from 3 healthy donors undergoing tumescence liposuction (age range, 32-52 years; 2 males and 1 female) who underwent surgery at the Shanghai General Hospital of Nanjing Medical University between January, 2018 and April, 2018. Clinical and biochemical examinations confirmed that these subjects did not suffer from acute inflammation, cancers, endocrine diseases or infectious diseases. The hADSCs were isolated as previously described (16). hADSCs were cultured in basal MSC culture medium (bM) [Dulbecco's modified Eagle's medium (DMEM), 10\% FCS penicillin/streptomycin, L-glutamine] (Lonza Group, Ltd.). The cells from the 3 donors were pooled together for all experiments and the experiments were performed 3 times. The present study was approved by the Ethics Committee of Shanghai General Hospital of Nanjing Medical University (IRB approval no. 2018-0032). Informed consent was obtained from all patients prior to study inclusion.

hADSC differentiation and staining analysis. hADSCs were grown in basal MSC culture medium at $37^{\circ} \mathrm{C}$ for 2 days, followed by differentiation into osteoblasts, adipocytes and chondrocytes. For osteogenic differentiation, the culture medium was exchanged for osteogenic differentiation medium (odM) [DMEM, $100 \mathrm{nM}$ dexamethasone, L-glutamine, $50 \mathrm{mM}$ ascorbate, $1 \%$ penicillin/streptomycin, mesangial cell growth supplement (MCGS) and $10 \mathrm{mM}$ $\beta$-lycerophosphate] (Lonza Group, Ltd.). The medium was changed every 3 days during the course of incubation (21 days) and the cells were harvested and analyzed at 7, 14 and 21 days. Cells cultured in bM medium served as a control. Alizarin Red S staining was performed to assess the occurrence of calcium phosphate deposition. Briefly, hADSCs were washed twice with PBS and fixed with $4 \%$ paraformaldehyde for $10 \mathrm{~min}$. The fixed cells were stained with $500 \mu \mathrm{l}$ of Alizarin Red solution (Sigma-Aldrich; Merck KGaA) for $5 \mathrm{~min}$ at room temperature. For adipogenic differentiation, the hADSCs were induced using the StemPro adipogenesis differentiation kit media (Gibco; Thermo Fisher Scientific, Inc.). The differentiation medium was changed every 3 days. After 21 days, Oil Red $\mathrm{O}$ staining was performed for the assessment of lipid/fatty acid droplets in adipocytes. Briefly, the fixed cells as above were stained with filtered $0.6 \mathrm{mg} / \mathrm{ml}$ Oil Red O solution for $30 \mathrm{~min}$ at room temperature. For chondrogenic differentiation, the hADSCs were induced using an MSC Chondrogenic Differentiation kit (Cyagen Bioscience, Inc.) according to the manufacturer's protocol. The differentiation medium was changed every 3 days. After 21 days, Alcian Blue staining was performed to assess the chondrogenic differentiation potential of hADSCs. Briefly, cells were rinsed in PBS and then fixed in methanol for $30 \mathrm{~min}$, following Alcian blue staining for $30 \mathrm{~min}$ at room temperature. All these images were viewed by a microscopy at x400 magnification.

Alkaline phosphatase (ALP) activity. The osteoblast phenotype was evaluated by determining ALP activity. Briefly, hADSCs were collected and lysed with RIPA buffer, and the total protein concentration was determined with Pierce BCA Protein Assay kit (Thermo Fisher Scientific). Relative ALP activity was normalized by the protein concentration. Then, the ALP activity of the hADSCs was detected with the Colorimetric Alkaline Phosphatase Assay kit (BioVision), according to the manufacturer's instructions.

Reverse transcription-quantitative polymerase chain reaction (RT-qPCR). miRNA was extracted from the hADSCs using an miRNeasy extraction kit (Qiagen, Inc.) according to the manufacturer's instructions. cDNA was synthesized using TaqMan $^{\text {TM }}$ MicroRNA Reverse Transcription kit (Applied Biosystems; Thermo Fisher Scientific, Inc.). To quantify the mRNA levels of runt-related transcription factor 2 (Runx2), osteopontin (OPN), osteocalcin (OCN) and bone sialoprotein (BSP), total RNA was isolated using TRIzol reagent (Invitrogen; Thermo Fisher Scientific, Inc.) according to the manufacturer's instructions. cDNA was synthesized using the PrimeScript RT reagent kit (Takara). Quantitative PCR was performed using a standard protocol from the SYBR-Green PCR kit (Toyobo) on an ABI 7500 thermocycler (Applied Biosystems; Thermo Fisher Scientific, Inc.). All reactions were performed in triplicate or duplicate. The relative levels of miRNAs and mRNA in cells were normalized to U6 and GAPDH, respectively. $95^{\circ} \mathrm{C}$ for $10 \mathrm{~min}$ followed by 45 cycles consisting of $95^{\circ} \mathrm{C}$ for $15 \mathrm{sec}, 60^{\circ} \mathrm{C}$ for $30 \mathrm{sec}$ and $68^{\circ} \mathrm{C}$ for $60 \mathrm{sec}$ were amplification conditions. The sequences of the primers were as follows: miR-29b forward, 5'-GGGGTAGCACCATTTGAA-3' and reverse, 5'-TGCGTG TCGTGGAGTC-3'; U6 forward, 5'-GCTTCGGCAGCACAT ATACTAAAAT-3' and reverse, 5'-CGCTTCACGAATTTG CGTGTCAT-3'; Runx2 forward, 5'-GTCTCACTGCCTCTC ACTTG-3' and reverse, 5'-CACACATCTCCTCCCTTCTG-3'; OCN forward, 5'-ACAGACAAGTCCCACACAGCAGC-3' and reverse, 5'-TGAAGGCTTTGTCAGACTCAGGGC-3'; BSP forward, 5'-GCCAGAGGAGCAATCACCAA-3' and reverse, 5'-CAGGCTGGAGGTTCACTGGT-3'; GAPDH forward, 5'-GTCTCACTGCCTCTCACTTG-3' and reverse, 5'-GTGGTGAAGACGCCAGTGGA-3'; OPN forward, 5'-TTG GCTTTGCAGTCTCCTGCGG-3' and reverse, 5'-AGGCAA GGCCGAACAGGCAAA-3' and GAPDH forward, 5'-CGA GCCACATCGCTCAGACA-3'. The relative expression of each gene was calculated using the $2^{-\Delta \Delta \mathrm{Cq}}$ method (17).

Microarray assay. Total RNA was isolated using a miRNAeasy mini kit (Qiagen, Inc.), followed by labeling and hybridization with the miRCURYTM LNA Array (v.16.0, Exiqon), which contained capture probes targeting all miRNAs for human, mouse and rats registered in the Sanger miRBase 21.0 database (www.mirbase.org/). The procedure and imaging processes were as described previously (18).

Cell transfection. Agomir-29b, antagomir-29b, phosphatase and tensin homolog (PTEN) overexpression plasmid and 
negative controls were all provided by RiboBio Co., Ltd. When the hADSCs in the 12-well plate grew to approximately $80 \%$ confluency, agomir-29b (0.1 nmol), antagomir-29b (0.1 nmol), or $2 \mu \mathrm{g}$ pcDNA-PTEN were transfected into the cells at $37^{\circ} \mathrm{C}$ for $24 \mathrm{~h}$, using Lipofectamine ${ }^{\circledR} 2000$ (Invitrogen; Thermo Fisher Scientific, Inc.).

Luciferase reporter assay. TargetScan Release 7.0 (http://targetscan.org/), Miranda (http://miranda.org.uk) and PicTar (https://pictar.mdc-berlin.de) were used to search for the putative targets of miR-34a.pGL3-PTEN wild-type (Wt) or pGL3-PTEN mutant type (mut) were co-transfected with agomir-29b (0.1 nmol), antagomir-29b (0.1 nmol) into 293T cells (ATCC) in 24 -well plates $\left(2 \times 10^{5} /\right.$ well) using Lipofectamine 2000 (Invitrogen). At $24 \mathrm{~h}$ post-transfection, the double luciferase activities were analyzed using the Dual-Luciferase Reporter Assay system (Promega Corp.). The pRL-TK plasmid (Promega Corp.) was used as a normalizing control.

Western blot analysis. Western blot analysis was performed as previously described (19). Briefly, the cells were lysed with RIPA buffer, and the total protein concentration was determined with Pierce BCA Protein Assay kit (Thermo Fisher Scientific). A total of $40 \mu \mathrm{g}$ protein samples were separated by $12 \%$ SDS-PAGE, and then transferred onto polyvinylidene difluoride (Millipore) membranes at $200 \mathrm{~mA}$ for $1 \mathrm{~h}$ on ice. After blocking with 5\% skim milk for $2 \mathrm{~h}$ at $4^{\circ} \mathrm{C}$ overnight, the membranes were incubated with the following primary antibodies: Anti-PTEN (cat. no. 9188, Cell Signaling Technology, Inc. 1:1,000), anti-Runx2 (cat. no. 12556, Cell Signaling Technology, Inc. 1:1,000), anti-OPN (cat. no. ab75285, Abcam, 1:1,000), anti-OCN (cat. no. ab13420, Abcam, 1:1,000), anti-BSP (cat. no. ab52128, Abcam, 1:1,000), anti-AKT (cat. no. 4691, Cell Signaling Technology, Inc. 1:1,000), phosphorylated-AKT (cat. no. 9611, Cell Signaling Technology, Inc. 1:1,000), glycogen synthase kinase (GSK)-3 $\beta$ (cat. no. 12456, Cell Signaling Technology, Inc. 1:1,000), phosphorylated-GSK-3 $\beta$ (Ser9) (cat. no. 8466, Cell Signaling Technology, Inc. 1:1,000), $\beta$-catenin (cat. no. 8480, Cell Signaling Technology, Inc. $1: 1,000$ ), phosphorylated- $\beta$-catenin (cat. no. 4176, Cell Signaling Technology, Inc. 1:1,000) at $4^{\circ} \mathrm{C}$ overnight. After washing with PBST, the membranes were incubated with horseradish peroxidase-conjugated secondary antibody (1:2,000; cat. no. 7074; Cell Signaling Technology, Inc.) for $1 \mathrm{~h}$ at room temperature. Finally, antibody labeling was detected by enhanced chemiluminescence (Thermo Fisher Scientific, Inc.). Band intensity was evaluated using ImageJ v1.48 U software (National Institutes of Health).

Statistical analysis. All data were presented as the means \pm standard deviation (SD). The comparisons among data were calculated by one-way ANOVA followed by Tukey's post hoc test by using SPSS software, version 13.0 (SPSS, Inc.). $\mathrm{P}<0.05$ was considered to indicate a statistically significant difference.

\section{Results}

miR-29b is upregulated during hADSC osteogenic differentiation. To assess the potential of hADSCs to differentiate into multiple lineages, several conventional analyses were conducted following the induction of osteogenesis, adipogenesis and chondrogenesis of hADSCs. As shown in Fig. 1A, hADSCs were able to differentiate into osteogenic cells (Alizarin Red S staining), adipocytes (Oil Red O staining) and chondrocytes (Alcian blue staining), which suggested that the hADSCs successfully differented into 3 different lineages. Alizarin Red S staining was performed to visualize calcium deposition in osteoblasts at 7,14 and 21 days following the induction of osteogenesis. It was found that calcium deposition in osteoblasts was significantly increased, and these promoting effects were time-dependent (Fig. 1B). Moreover, the osteoblast phenotype was evaluated by determining ALP activity. The results revealed that ALP activity was also increased in a time-dependent manner (Fig. 1C). Several osteogenic markers, (Runx2 and BSP as early marker genes), (OPN and $\mathrm{OCN}$ as late marker genes) were also examined at the genomic level. As expected, the expression levels of these osteogenic markers were markedly increased in a time-dependent manner (Fig. 1D-G), suggesting that the osteogenic differentiation of hADSCs was induced successfully.

Previous studies have suggested that miRNAs play a role in osteogenic differentiation $(20,21)$. In the present study, using microarray assay, it was found that the levels of 24 miRNAs were increased and those of 14 miRNAs were decreased in the differentiated cells compared with the undifferentiated control cells (Fig. 1H). Of the upregulated miRNAs, miR-29b expression displayed the most alteration in this array. Of relevance, miR-29b has been identified as a key regulator of the osteogenic differentiation of MSCs by targeting anti-osteogenic factors or modulating bone extracellular matrix proteins in a mineralogenic cell system (ABSa15) or in MC3T3 cells $(22,23)$. However, whether miR-29b affects the osteogenic differentiation of hADSCs remains unclear. To further verify the expression of miR-29b, the expression of miR-29b was quantified by RT-qPCR at different time points during the osteogenesis process. As shown in Fig. 1I, the results revealed that miR-29b expression was increased in a time-dependent manner compared with the undifferentiated control cells. These results suggest that miR-29b may play an important role in the osteogenic differentiation of hADSCs.

Overexpression of miR-29b promotes the osteogenic differentiation of hADSCs. To determine whether miR-29b affects the osteogenic differentiation of hADSCs, the hADSCs was transfected with agomir-29b, agomir-negative control (agomir NC), antagomir-29b and antagomir NC during osteogenic differentiation. As shown in Fig. 2A, miR-29b expression was significantly increased (or decreased) in the hADSCs following agomir-29b (or antagomir-29b) transfection, compared with the agomir (or antagomir) NC group. At $16 \mathrm{~h}$ after transfection, the medium was exchanged for osteogenic medium and the effects of miR-29b on the hADSCs induced to differentiate into osteoblasts were evaluated. It was observed that the overexpression of miR-29b significantly increased the mineralization of the extracellular matrix and ALP activity, while miR-29b inhibition produced the opposite results (Fig. 2B and C). Additionally, supporting the above-mentioned observations, agomir-29b increased the expression of bone-specific markers, such as Runx2, BSP, OPN 


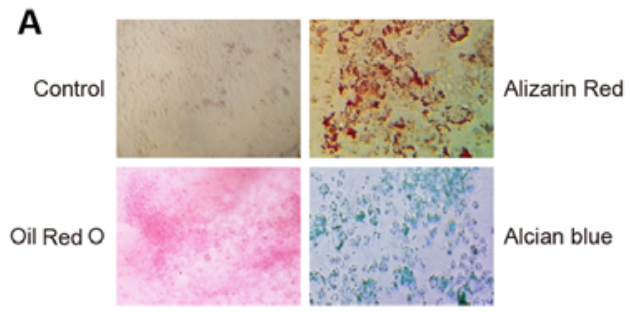

B

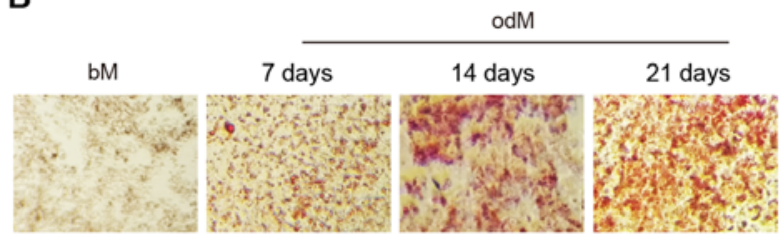

C

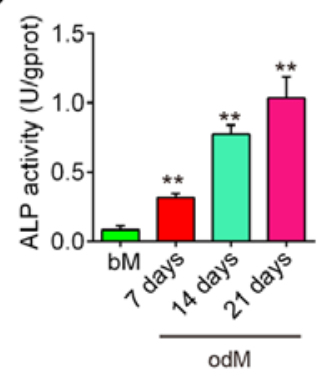

G

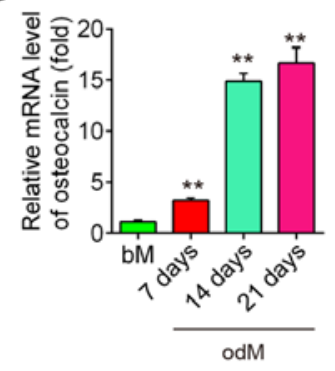

D

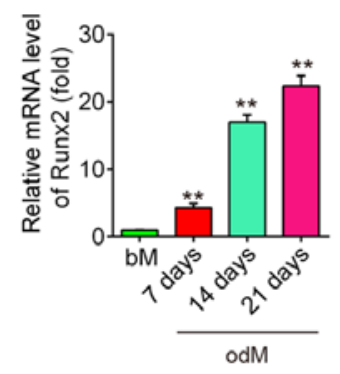

H

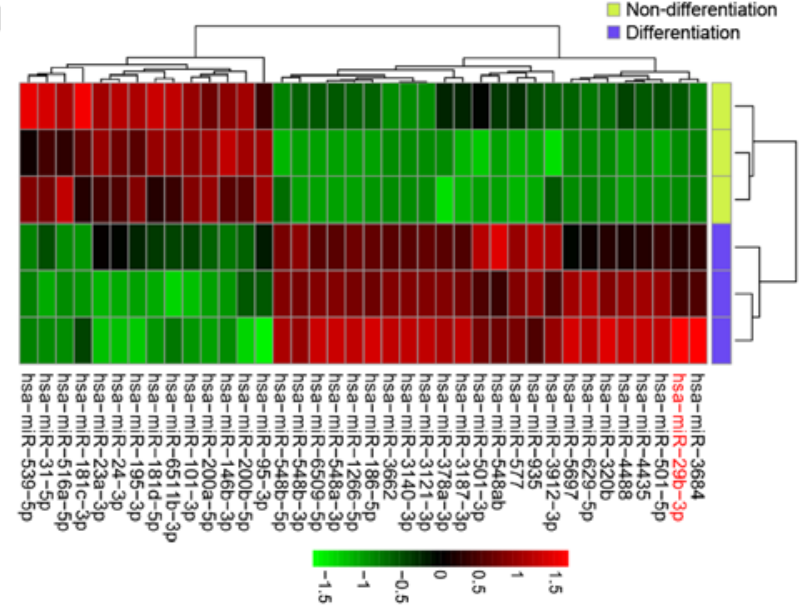

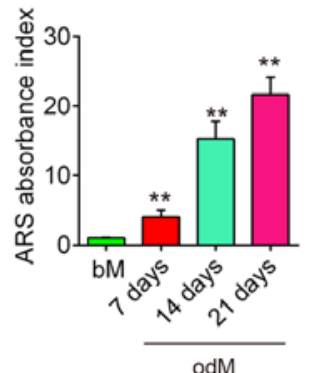

F

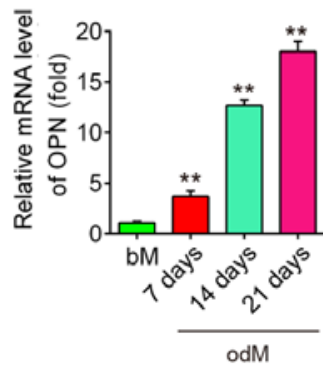

I

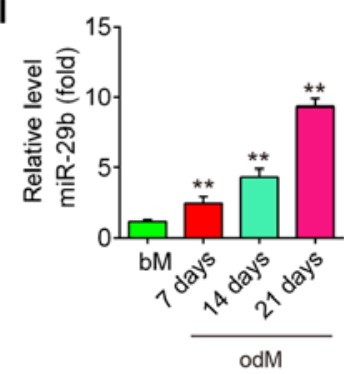

Figure 1. miR-29b is downregulated during hADSC differentiation. (A) Photograph morphology of hADSCs following Oil Red O, Alizarin Red S or Alcian Blue staining following 21 days of culture in normal, adipogenic, osteogenic or chondrogenic differentiation medium. Magnification, x200. (B) Alizarin Red S staining at 7, 14 and 21 days following osteogenic differentiation. (C) Quantitative assessment of ALP activity by colorimetric alkaline phosphatase assay at 7, 14 and 21 days after osteogenic differentiation. RT-qPCR analysis was used to assess the mRNA expression levels of the osteogenic differentiation markers (D) Runx2, (E) BSP, (F) OPN and (G) osteocalcin at 7, 14 and 21 days following osteogenic differentiation. (H) Heatmap of miRNA profiles represents the differentially expressed miRNAs between differentiated and undifferentiated hADSCs. Green indicates low expression levels; red indicates high expression levels. (I) RT-qPCR analysis was used to assess the expression levels of miR-29b on days 7, 14 and 21 post-induction. Data are represented as the means \pm SD of 3 independent experiments. ${ }^{* *} \mathrm{P}<0.01$ vs. bM group. hADSC, human adipose derived mesenchymal stem cell; Runx2, runt-related transcription factor 2; BSP, bone sialoprotein; OPN, osteopontin; bM, basal mesenchymal stem cell culture medium.

and OCN at the mRNA and protein level, whereas the expression of bone-specific markers was significantly suppressed by antagomir-29b infection (Fig. 2D-H). More importantly, the effects of miR-29b on the tri-lineage differentiation potential of hADSCs were examined. As shown in Fig. 2I, miR-29b upregulation led to a significant increase in the adipogenic and chondrogenic differentiation of hADSCs, while miR-29b downregulation exerted an opposite effect, which is consistent with the findings of a previous study (24). These data suggest that miR-29b plays a regulatory role in the adipogenic and chondrogenic differentiation of hADSCs. Notably, previous studies have paid increasing attention to the osteogenic differentiation of hADSCs as an effective treatment for musculoskeletal disorders, such as osteoarthritis (OA) (25) and rheumatoid arthritis (RA) (26). Therefore, the present study focused on the progression of the osteogenic differentiation of hADSCs regulated by miR-26. In the future, the authors aim to further investigate the mechanisms underlying the regulation of the adipogenic and chondrogenic differentiation of hADSCs by $\operatorname{miR}-29 b$.

PTEN is a direct target of miR-29b. Through bioinformatics online programs, such as TargetScan, miRanda and PicTar, PTEN, a negative regulator of the AKT/mTOR signaling pathway, was found to have a putative target site of miR-29b in its 3'-UTR (Fig. 3A). To validate the possibility that PTEN was directly targeted by miR-29b, a luciferase reporter assay was performed. Luciferase reporter assay revealed that miR-29b mimics markedly inhibited the luciferase activity in the constructs containing the wild-type binding site of PTEN-3'UTR, and that the miR-29b inhibitor caused an increased luciferase activity. However, the luciferase activity 
A
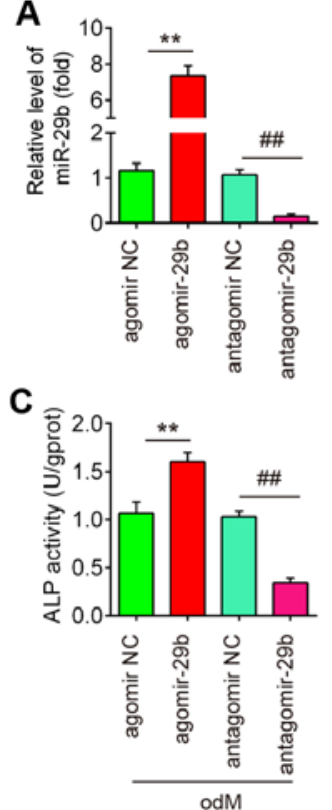

G

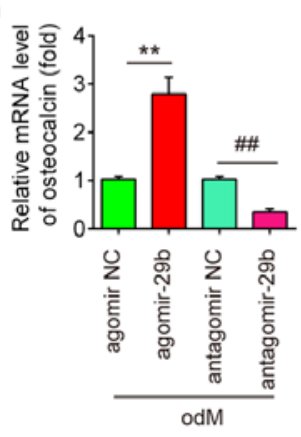

B
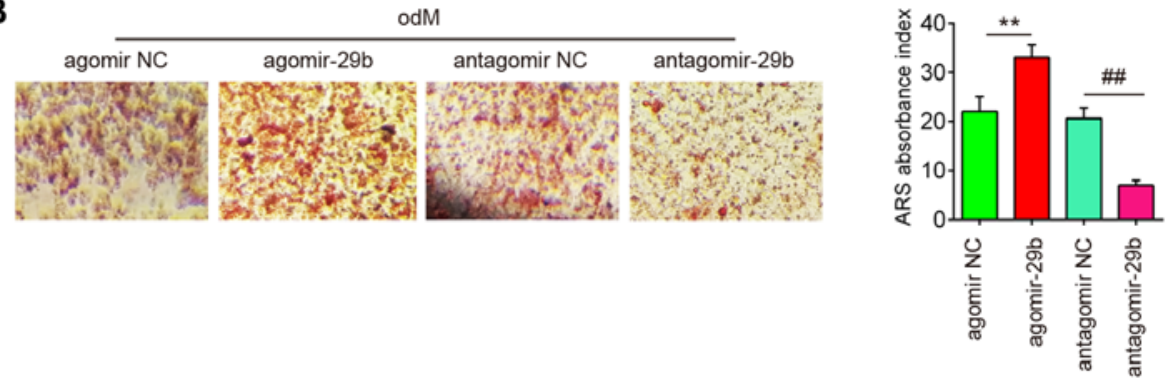

D

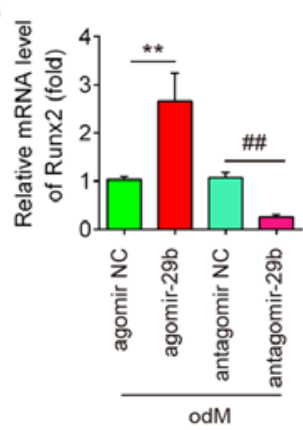

E

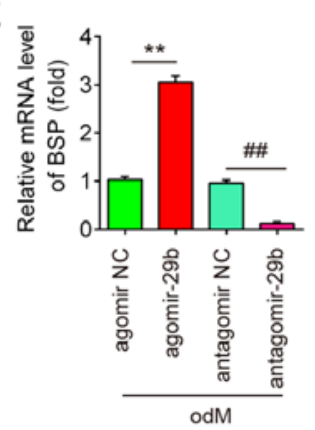

F

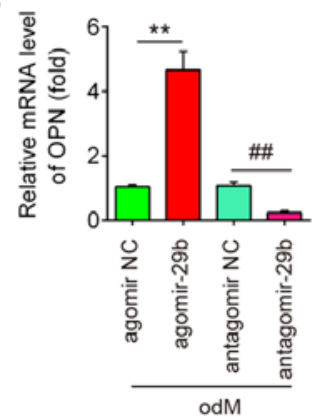

H

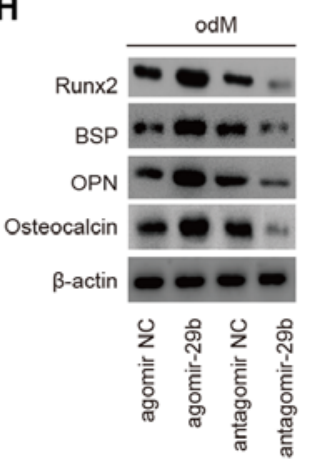

I

odM

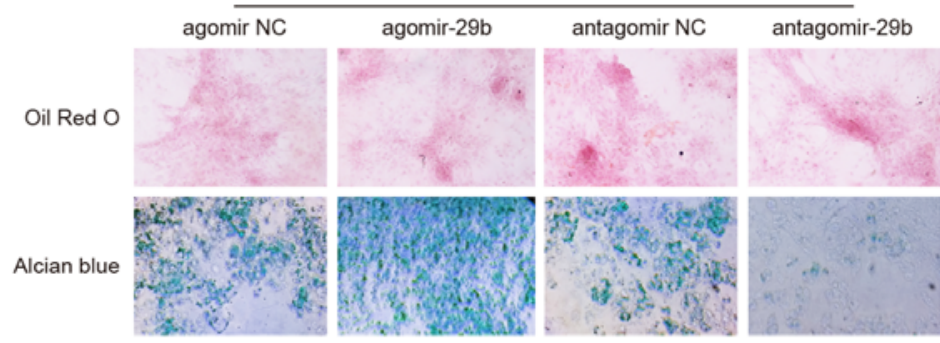

Figure 2. Overexpression of miR-29b promotes the osteogenic differentiation of hADSCs. hADSCs were transfected with agomir-29b, agomir NC, antagomir-29b and antagomir NC. At $48 \mathrm{~h}$ following transfection, cells were induced with differentiation medium. (A) RT-qPCR analysis was used to assess the expression levels of miR-29b $48 \mathrm{~h}$ after transfection. (B) Osteogenic differentiation was determined by staining with Alizarin Red S on day 21 post-induction. (C) Quantitative assessment of ALP activity by colorimetric alkaline phosphatase assay on day 21 post-induction. RT-qPCR analysis was used to assess the mRNA expression levels of the osteogenic differentiation markers (D) Runx2, (E) BSP, (F) OPN and osteocalcin (G) on day 21 post-induction. (H) Western blot analysis was used to assess the protein expression levels of the osteogenic differentiation markers (Runx2, BSP, OPN and osteocalcin). (I) Photograph morphology of hADSCs following Oil Red O, Alizarin Red S or Alcian blue staining after 21 days of culture in normal, adipogenic, osteogenic or chondrogenic differentiation medium. Magnification, $\mathrm{x} 200$. Data were represented as the means \pm SD of 3 independent experiments. ${ }^{* *} \mathrm{P}<0.01$ vs. agomir $\mathrm{NC}$ group; ${ }^{\#} \mathrm{P}<0.01$ vs. antagomir NC group. hADSC, human adipose derived mesenchymal stem cell; Runx2, runt-related transcription factor 2; BSP, bone sialoprotein; OPN, osteopontin.

of the constructs containing the mutant binding site exhibited no difference upon miR-29b expression compared with the negative control (Fig. 3B). Subsequently, to further validate the suppressive effect of miR-29b on PTEN, the expression of PTEN was measured in the hADSCs by western blot analysis. As shown in Fig. 3C, the PTEN levels were significantly downregulated following transfection with agomir-29b, whereas they were increased following transfection with antagomir-29b (Fig. 3C). In addition, the expression of PTEN decreased in a time-dependent manner during the osteogenic differentiation of hADSCs, as determined by western blot analysis (Fig. 3D). These results indicated that miR-29b can bind to PTEN through specific sites and can downregulate PTEN expression during osteogenic differentiation.

miR-29b activates the AKT/ $\beta$-catenin signaling pathway through the downregulation of PTEN expression. PTEN is an important upstream regulator of the AKT signaling pathway that has been implicated as an important regulator of osteoblast differentiation in MSCs $(27,28)$. However, the association between miR-29b and the PTEN/Akt pathway in the osteogenic differentiation of hADSCs remains unclear. As shown in Fig. 4, the introduction of agomir-29b into the hADSCs significantly suppressed the expression of PTEN, but promoted p-AKT protein expression. As is already known, the AKT/GSK-3 $\beta / \beta$-catenin signaling pathway is important for MSC differentiation, including osteogenic differentiation $(6,29)$. Therefore, the present study further investigated whether miR-29b affects the AKT/GSK-3 $\beta / \beta$-catenin signaling pathway during the osteogenic differentiation of hADSCs. As shown in Fig. 4, the overexpression of miR-29b in the hADSCs induced the expression of phospho-GSK3 $\beta$ and phospho- $\beta$-catenin compared with the cells infected with agomir-NC. These results suggest that miR-29b 


\section{A}

Position 1741-1747 of PTEN 3' UTR

$\begin{array}{rc}\text { hsa-miR-29b-3p } & \text { 3'...UUGUGACUAAAGUUUACCACGAU... 5' } \\ & \mathbf{:}::::: \\ \text { PTEN wt } & \text { 5'...UUUUCAAUAACUUAUUGGUGCUG... 3' } \\ \text { PTEN mut } & \text { 5'...UUUUCAAUAACUUAUACCAGAGG... 3' }\end{array}$
B

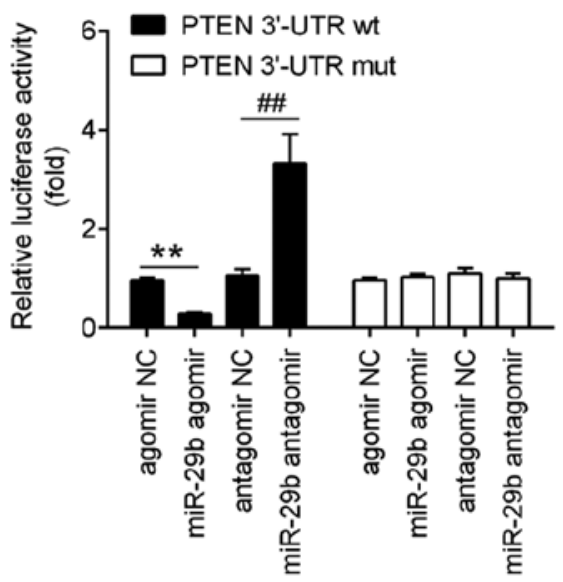

C

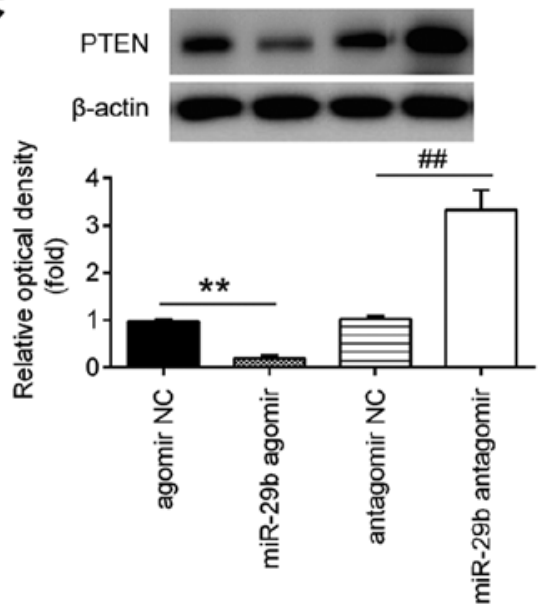

D

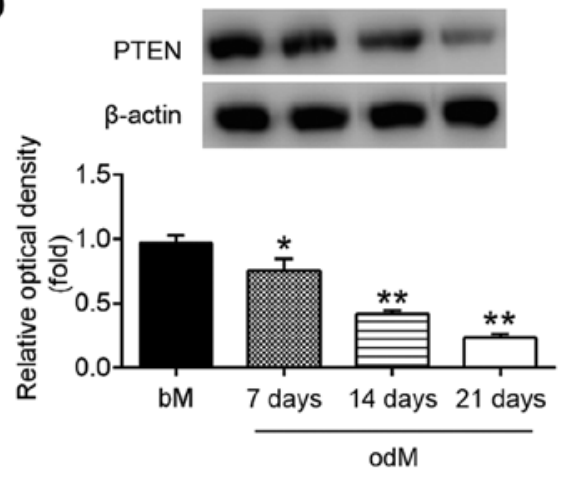

Figure 3. PTEN is a direct target of miR-29b. (A) Putative binding sites of between miR-29b and PTEN. (B) hADSCs were co-transfected with luciferase reporter constructs containing wt or mut PTEN 3'-UTR and agomir-29b, agomir NC, antagomir-29b, and antagomir NC and luciferase activity was then detected $(\mathrm{n}=3)$. Data are represented as the means $\pm \mathrm{SD}$ of 3 independent experiments. ${ }^{* *} \mathrm{P}<0.01$ vs. agomir NC group; ${ }^{\# \#} \mathrm{P}<0.01$ vs. antagomir NC group. (C) Western blot analysis was used to assess the protein expression levels of PTEN in hADSCs following transfection with agomir-29b, agomir NC, antagomir-29b, and antagomir NC $(\mathrm{n}=3)$. Data are represented as the means $\pm \mathrm{SD}$ of 3 independent experiments. ${ }^{* * *} \mathrm{P}<0.01$ vs. agomir NC group; ${ }^{\# \#} \mathrm{P}<0.01$ vs. antagomir NC group. (D) Western blot analysis was used to assess the protein expression levels of PTEN in hADSCs at days 7, 14 and 21 post-induction. Data are represented as the means \pm SD of 3 independent experiments. ${ }^{*} \mathrm{P}<0.05,{ }^{* * *} \mathrm{P}<0.01 \mathrm{vs}$. bM group. PTEN, phosphatase and tensin homolog; hADSC, human adipose derived mesenchymal stem cell; $\mathrm{bM}$, basal mesenchymal stem cell culture medium.

promotes the osteogenic differentiation of hADSCs via the PTEN/AKT/GSK-3 $\beta / \beta$-catenin pathway.

PTEN reverses the promoting effects of $m i R-29 b$ on the osteogenic differentiation of hADSCs. To further investigate whether PTEN is involved in the miR-29b-mediated osteogenic differentiation of hADSCs, the hADSCs were co-transfected with agomir-29b and pcDNA-PTEN during osteogenic differentiation. It was found that the protein expression of PTEN was significantly increased in the hADSCs when they were transfected with the pcDNA-PTEN plasmid (Fig. 5A). As was expected, the protein expression of PTEN was also significantly increased in the miR-29b-overexpressing hADSCs when they were transfected with the pcDNA-PTEN plasmid (Fig. 5B). Subsequently, the ALP activity, the changes in matrix mineralization and the levels of osteogenic marker genes were assessed by ALP staining, Alizarin Red S staining and RT-qPCR assays, respectively. The results revealed that the upregulation of miR-29b by agomir-29b significantly enhanced the ALP activity and the mineralization of extracellular matrix, and led to an increase in Runx2, OPN, OCN and BSP mRNA expression, whereas the promoting effects of agomir-29b were reversed by the overexpression of PTEN (Fig. 5C-H). Collectively, these data suggest that miR-29b promotes the osteogenic differentiation of hADSCs by targeting PTEN.

\section{Discussion}

In the present study, miR-29b expression was found to be significantly upregulated during the osteogenic differentiation of hADSCs. Further experiments demonstrated that miR-29b promoted the osteogenic differentiation of hADSCs through the activation of the $\mathrm{AKT} / \beta$-catenin signaling pathway via the downregulating PTEN. These findings provide novel insight into the mechanisms underlying osteogenic differentiation and contribute to development of therapies for bone defects by targeting miRNAs.

Dozens of miRNAs have been demonstrated to modulate numerous biological processes, including the differentiation of hADSCs $(30,31)$. For example, Huang et al demonstrated that the overexpression of miR-22 promoted the osteogenic 
A

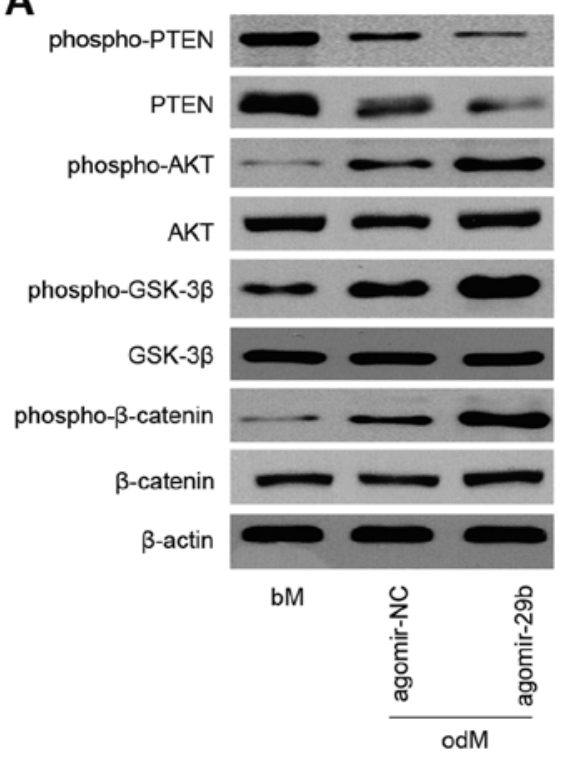

B

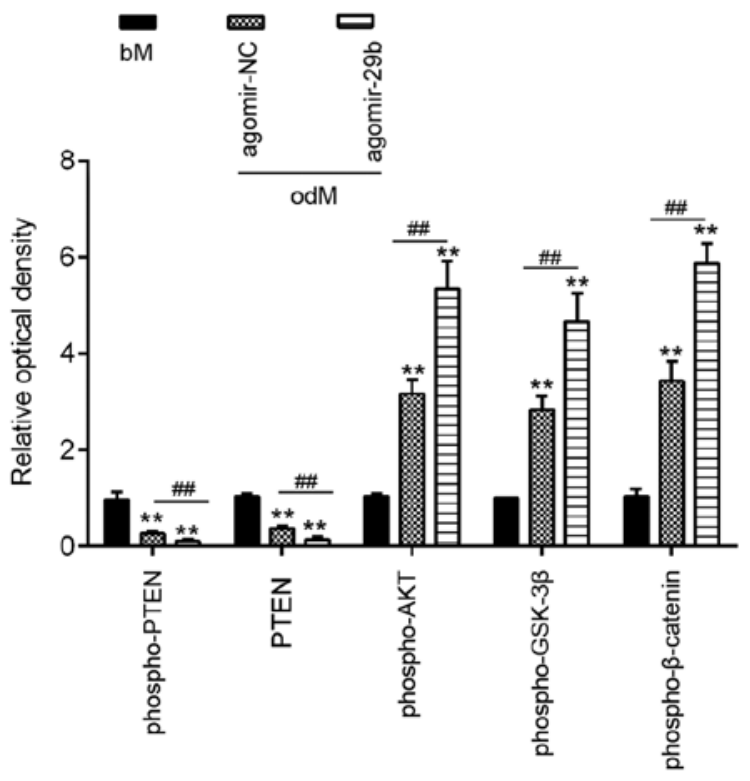

Figure 4. miR-29b activates the AKT/ $\beta$-catenin signaling pathway by downregulating PTEN expression. hADSCs were transfected with agomir-29b and agomir NC, following the induction of osteogenic differentiation. (A) Western blot analysis was used to assess the protein expression levels of PTEN, phospho-AKT, AKT, phospho-GSK-3 $\beta$, GSK-3 $\beta$, phospho- $\beta$-catenin and $\beta$-catenin. $\beta$-actin was used as an internal control. (B) The bands were semi-quantitatively analyzed using ImageJ software, normalized to $\beta$-actin density. Data represent the means \pm SD of 3 independent experiments. ${ }^{* *} \mathrm{P}<0.01 \mathrm{vs}$. bM group, ${ }^{\# \#} \mathrm{P}<0.01 \mathrm{vs}$. agomir NC group. PTEN, phosphatase and tensin homolog; hADSC, human adipose derived mesenchymal stem cell; GSK-3 $\beta$, glycogen synthase kinase $3 \beta$.

A

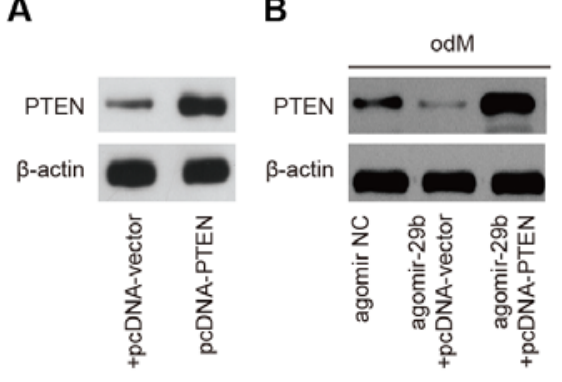

C

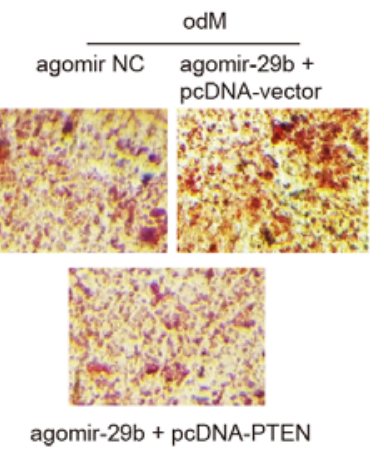

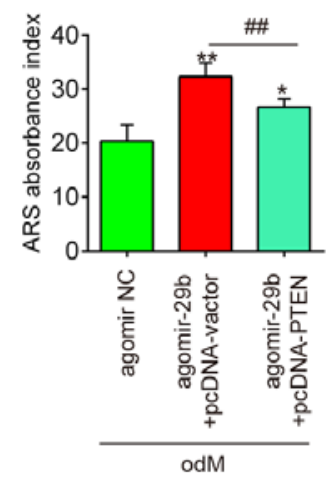

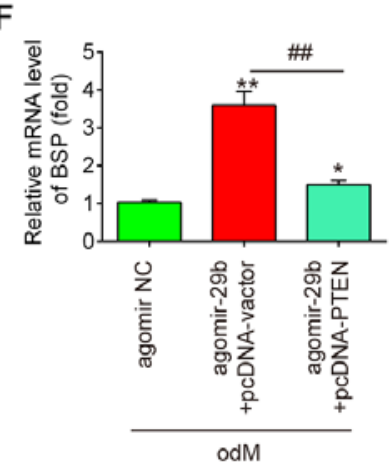

$\mathbf{G}$

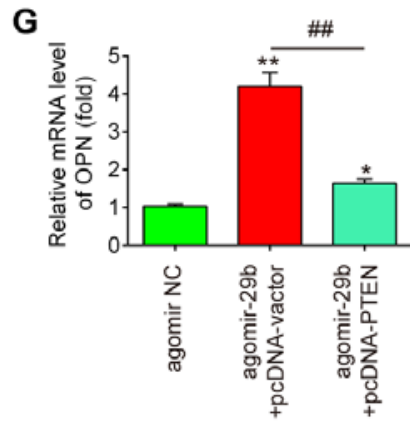

odM

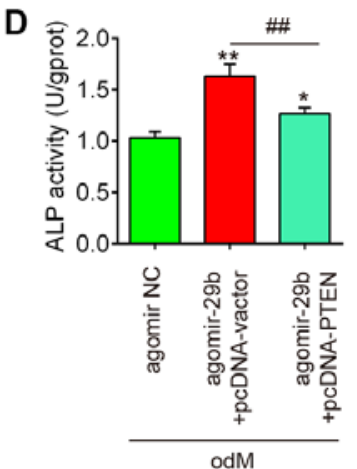

H

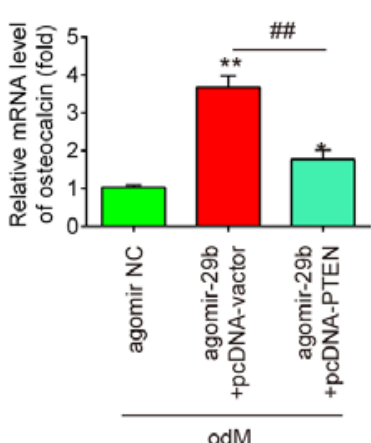

Figure 5. PTEN reverses the promoting effects of miR-29b on the osteogenic differentiation of hADSCs. hADSCs were co-transfected with agomir-29b and pcDNA-PTEN, following induction of osteogenic differentiation. (A) The expression of PTEN protein was measured in hADSCs when the pcDNA-PTEN plasmid was transfected by western blot analysis. (B) Western blot analysis was used to assess the protein expression levels of PTEN in hADSCs following transfection with agomir-29b and pcDNA-PTEN. (C) Osteogenic differentiation was determined by staining with Alizarin Red S staining on day 21 post-induction. Shown is a representative example of 3 independent experiments. (D) Quantitative assessment of ALP activity by colorimetric alkaline phosphatase assay on day 21 post-induction. RT-qPCR analysis was used to assess the mRNA expression levels of the osteogenic differentiation markers (E) Runx2, (F) BSP, (G) OPN and osteocalcin $(\mathrm{H})$ on day 21 post-induction. Data represent the means $\pm \mathrm{SD}$ of 3 independent experiments. ${ }^{*} \mathrm{P}<0.05$, ${ }^{* * *} \mathrm{P}<0.01$ vs. agomir NC group, ${ }^{\# \prime} \mathrm{P}<0.01$ vs. agomir-29b + pcDNA-vector group. PTEN, phosphatase and tensin homolog; hADSC, human adipose derived mesenchymal stem cell.

differentiation and inhibited the adipogenic differentiation of hADSCs by suppressing HDAC6 protein expression (32).
Zeng et al revealed that miR-100 targeting bone morphogenetic protein receptor type II (BMPR2) inhibited the 
osteogenic differentiation of hADSCs (33). In the present study, using microarray assay, miR-29b was found to be significantly upregulated during the osteogenic induction of hADSCs, and this effect was time-dependent. Previous studies have indicated that miR-29b is aberrantly expressed during the osteoblast differentiation of murine calvaria-derived preosteoblasts (MC3T3) and acts as a positive regulator of the osteogenic differentiation of MC3T3 cells $(15,22,23)$. However, these studies were performed on the MC3T3 osteoblast cell line. Improvements in the knowledge of the characteristics of miRNAs in osteogenesis provide an important step for their application in translational investigations of bone tissue engineering and bone disease. Therefore, the present study determine whether miR-29b plays a similar role in the osteogenic differentiation of hADSCs and further examined its potential molecular mechanisms. It was found that the overexpression of miR-29b promoted the mineralized deposition and ALP activity of hADSCs, whereas the inhibition of miR-29b suppressed these processes. The expression profiles of osteogenic markers (Runx2, BSP, OPN and OCN) in the hADSCs further confirmed this finding. These data indicate that miR-29b functions as a positive regulator of hADSC osteogenesis.

Various studies have indicated that miRNAs regulate MSC osteogenesis by acting on the corresponding genes that regulate osteogenic differentiation $(1,34)$. PTEN has been previously reported to function as a key transcription factor on controlling osteoblast functions (35-37). For example, Shen et al found that the loss of PTEN in bone marrow-derived MSCs (BMSCs) led to a decreased osteogenic differentiation potential by increasing the proliferative capability (38). Liu et al demonstrated that PTEN deletion mutants increased osteoblast activity and bone mineral density (39). In the present study, using bioinformatic analysis via TargetScan and miRanda, PTEN was identified as one of the potential targets of miR-29b, which is consistent with the findings of a previous study (40). It was also indicated that miR-29b suppressed the expression of PTEN during the osteogenic differentiation of hADSCs. Therefore, it was hypothesized that the miR-29b/PTEN axis may play a key role in hADSC osteogenesis. As was expected, it was demonstrated that the promoting effects of agomir-29b on ALP activity, matrix mineralization and the levels of osteogenic marker genes were reversed by the overexpression of PTEN. These data suggest that miR-29b promotes the osteogenic differentiation of hADSCs by suppressing the expression of PTEN.

To date, a variety of signaling pathways that regulate osteogenic differentiation have been discovered. Among these, the AKT $/ \beta$-catenin pathway is considered to be a critical negative regulator of the osteogenic differentiation of MCS $(41,42)$. Previous studies have demonstrated that the activation of AKT is sufficient to activate the $\beta$-catenin signaling pathway, whereupon $\beta$-catenin in itself was previously demonstrated to potentiate osteogenesis (43). Given the fact that PTEN can restrict AKT $(44,45)$, it is possible that miR-29b regulates the osteogenic differentiation of hADSCs via the AKT/ $\beta$-catenin pathway. In the present study, it was proven that the upregulation of miR-29b promoted the expression levels of phospho-AKT, phospho-GSK $3 \beta$ and phospho- $\beta$-catenin, suggesting that miR-29b can activate the AKT/GSK-3 $\beta / \beta$-catenin signaling pathway. Therefore, these results indicate that miR-29b can activate the AKT/mTOR pathway by suppressing PTEN expression, thereby promoting the osteogenic differentiation of hADSCs.

In conclusion, the present study demonstrated that miR-29b promoted the osteogenic differentiation of hADSCs, at least partly through the PTEN/AKT/GSK-3 $\beta / \beta$-catenin signaling pathway. These observations provide preclinical data supporting the potential application of hADSCs engineered with miR-29b in curing bone-related diseases.

\section{Acknowledgements}

Not applicable.

\section{Funding}

No funding was received.

\section{Availability of data and materials}

All data generated or analyzed during the present study are included in this published article.

\section{Authors' contributions}

TX and SD performed the experiments, contributed to data analysis and wrote the manuscript. TX and SD analyzed the data. JT conceptualized the study design, contributed to data analysis and experimental materials. All authors read and approved the final manuscript.

\section{Ethics approval and consent to participate}

The present study was approved by the Ethics Committee of Shanghai General Hospital of Nanjing Medical University (IRB approval no. 2018-0032). Informed consent was obtained from all patients prior to study inclusion.

\section{Patient consent for publication}

Not applicable.

\section{Competing interests}

The authors declare that they have no competing interests.

\section{References}

1. Deng Y, Zhou H, Zou D, Xie Q, Bi X, Gu P and Fan X: The role of miR-31-modified adipose tissue-derived stem cells in repairing rat critical-sized calvarial defects. Biomaterials 34: 6717-6728, 2013.

2. Lin CY, Chang YH, Li KC, Lu CH, Sung LY, Yeh CL, Lin KJ, Huang SF, Yen TC and Hu YC: The use of ASCs engineered to express BMP2 or TGF- $\beta 3$ within scaffold constructs to promote calvarial bone repair. Biomaterials 34: 9401-9412, 2013.

3. Locke M, Feisst V and Dunbar PR: Concise review: Human adipose-derived stem cells: Separating promise from clinical need. Stem Cells 29: 404-411, 2011.

4. Gadkari R, Zhao L, Teklemariam T and Hantash BM: Human embryonic stem cell derived-mesenchymal stem cells: An alternative mesenchymal stem cell source for regenerative medicine therapy. Regen Med 9: 453-465, 2014. 
5. Song F, Jiang D, Wang T, Wang Y,Lou Y,Zhang Y,MaHand Kang Y: Mechanical stress regulates osteogenesis and adipogenesis of rat mesenchymal stem cells through PI3K/Akt/GSK-3 $\beta / \beta$-catenin signaling pathway. Biomed Res Int 2017: 6027402, 2017.

6. Wu X, Zheng S, Ye Y, Wu Y, Lin K and Su J: Enhanced osteogenic differentiation and bone regeneration of poly(lactic-co-glycolic acid) by graphene via activation of PI3K/Akt/GSK-3 $\beta / \beta$-catenin signal circuit. Biomater Sci 6: 1147-1158, 2018.

7. Saidak Z, Le Henaff C, Azzi S, Marty C, Da Nascimento S, Sonnet $\mathrm{P}$ and Marie PJ: Wnt/ $\beta$-catenin signaling mediates osteoblast differentiation triggered by peptide-induced $\alpha 5 \beta 1$ integrin priming in mesenchymal skeletal cells. J Biol Chem 290 6903-6912, 2015.

8. Chen JC and Jacobs CR: Mechanically induced osteogenic lineage commitment of stem cells. Stem Cell Res Ther 4: 107 2013 .

9. Ambros V: The functions of animal microRNAs. Nature 431: 350-355, 2004

10. Bartel DP: MicroRNAs: Genomics, biogenesis, mechanism, and function. Cell 116: 281-297, 2004.

11. Luzi E, Marini F, Sala SC, Tognarini I, Galli G and Brandi ML: Osteogenic differentiation of human adipose tissue-derived stem cells is modulated by the miR-26a targeting of the SMAD1 transcription factor. J Bone Miner Res 23: 287-295, 2008.

12. Li J, Hu C, Han L, Liu L, Jing W, Tang W, Tian W and Long J: MiR-154-5p regulates osteogenic differentiation of adipose-derived mesenchymal stem cells under tensile stress through the Wnt/PCP pathway by targeting Wnt11. Bone 78: 130-141, 2015.

13. Kim YJ, Bae SW, Yu SS, Bae YC and Jung JS: miR-196a regulates proliferation and osteogenic differentiation in mesenchymal stem cells derived from human adipose tissue. J Bone Miner Res 24: 816-825, 2009.

14. Chen S, Zheng Y, Zhang S, Jia L and Zhou Y: Promotion effects of miR-375 on the osteogenic differentiation of human adipose-derived mesenchymal stem cells. Stem Cell Reports 8 773-786, 2017

15. Peng S, Gao D, Gao C, Wei P, Niu M and Shuai C: MicroRNAs regulate signaling pathways in osteogenic differentiation of mesenchymal stem cells (Review). Mol Med Rep 14: 623-629, 2016.

16. Boquest AC, Shahdadfar A, Brinchmann JE and Collas P: Isolation of stromal stem cells from human adipose tissue. Methods Mol Biol 325: 35-46, 2006.

17. Livak KJ and Schmittgen TD: Analysis of relative gene expression data using real-time quantitative PCR and the 2(-Delta Delta C(T)) method. Methods 25: 402-408, 2001.

18. Mei LL, Wang WJ, Qiu YT, Xie XF, Bai J and Shi ZZ: miR-125b-5p functions as a tumor suppressor gene partially by regulating HMGA2 in esophageal squamous cell carcinoma. PLoS One 12: e0185636, 2017

19. Zhang MY, Lin J and Kui YC: MicroRNA-345 suppresses cell invasion and migration in non-small cell lung cancer by directly targeting YAP1. Eur Rev Med Pharmacol Sci 23: 2436-2443, 2019.

20. Fang S, Deng Y, Gu P and Fan X: MicroRNAs regulate bone development and regeneration. Int J Mol Sci 16: 8227-8253, 2015

21. Tang P, Xiong Q, Ge W and Zhang L: The role of microRNAs in osteoclasts and osteoporosis. RNA Biol 11: 1355-1363, 2014.

22. Roberto VP, Tiago DM, Silva IA and Cancela ML: MiR-29a is an enhancer of mineral deposition in bone-derived systems. Arch Biochem Biophys 564: 173-183, 2014.

23. Li Z, Hassan MQ, Jafferji M, Aqeilan RI, Garzon R, Croce CM, van Wijnen AJ, Stein JL, Stein GS and Lian JB: Biological functions of miR-29b contribute to positive regulation of osteoblast differentiation. J Biol Chem 284: 15676-15684, 2009.

24. Mayer U, Benditz A and Grässel S: miR-29b regulates expression of collagens I and III in chondrogenically differentiating BMSC in an osteoarthritic environment. Sci Rep 7: 13297, 2017.

25. Wang W and Cao W: Treatment of osteoarthritis with mesenchymal stem cells. Sci China Life Sci 57: 586-595, 2014.

26. Baharlou R, Ahmadi-Vasmehjani A, Faraji F, Atashzar MR, Khoubyari M, Ahi S, Erfanian S and Navabi SS: Human adipose tissue-derived mesenchymal stem cells in rheumatoid arthritis: Regulatory effects on peripheral blood mononuclear cells activation. Int Immunopharmacol 47: 59-69, 2017.

27. Meng YB, Li X, Li ZY, Zhao J, Yuan XB, Ren Y, Cui ZD, Liu YD and Yang XJ: microRNA-21 promotes osteogenic differentiation of mesenchymal stem cells by the PI3K/ $\beta$-catenin pathway. J Orthop Res 33: 957-964, 2015.
28. Shen GY, Ren H, Huang JJ, Zhang ZD, Zhao WH, Yu X, Shang Q, Qiu T, Zhang YZ, Tang JJ, et al: Plastrum testudinis extracts promote BMSC proliferation and osteogenic differentiation by regulating let-7f-5p and the TNFR2/PI3K/AKT signaling pathway. Cell Physiol Biochem 47: 2307-2318, 2018

29. Xie Q, Wang Z, Zhou H, Yu Z, Huang Y, Sun H, Bi X, Wang Y, Shi W, Gu P and Fan X: The role of miR-135-modified adipose-derived mesenchymal stem cells in bone regeneration. Biomaterials 75: 279-294, 2016.

30. Hu R, Li H, Liu W, Yang L, Tan YF and Luo XH: Targeting miRNAs in osteoblast differentiation and bone formation. Expert Opin Ther Targets 14: 1109-1120, 2010.

31. Cho HH, Shin KK, Kim YJ, Song JS, Kim JM, Bae YC, Kim CD and Jung JS: NF-kappaB activation stimulates osteogenic differentiation of mesenchymal stem cells derived from human adipose tissue by increasing TAZ expression. J Cell Physiol 223: 168-177, 2010.

32. Huang S, Wang S, Bian C, Yang Z, Zhou H, Zeng Y, Li H, Han Q and Zhao RC: Upregulation of miR-22 promotes osteogenic differentiation and inhibits adipogenic differentiation of human adipose tissue-derived mesenchymal stem cells by repressing HDAC6 protein expression. Stem Cells Dev 21: 2531-2540, 2012.

33. Zeng Y, Qu X, Li H, Huang S, Wang S, Xu Q, Lin R, Han Q, Li J and Zhao RC: MicroRNA-100 regulates osteogenic differentiation of human adipose-derived mesenchymal stem cells by targeting BMPR2. FEBS Lett 586: 2375-2381, 2012.

34. Wei J, Shi Y, Zheng L, Zhou B, Inose H, Wang J, Guo XE, Grosschedl R and Karsenty G: miR-34s inhibit osteoblast proliferation and differentiation in the mouse by targeting SATB2. J Cell Biol 197: 509-521, 2012.

35. Nielsen-Preiss SM, Silva SR and Gillette JM: Role of PTEN and Akt in the regulation of growth and apoptosis in human osteoblastic cells. J Cell Biochem 90: 964-975, 2003.

36. Zhang Y, Zhang L, Yan M and Zheng X: Inhibition of phosphatidylinositol 3-kinase causes cell death in rat osteoblasts through inactivation of Akt. Biomed Pharmacother 61: 277-284, 2007.

37. Kawamura N, Kugimiya F, Oshima Y, Ohba S, Ikeda T, Saito T, Shinoda Y, Kawasaki Y, Ogata N, Hoshi K, et al: Akt1 in osteoblasts and osteoclasts controls bone remodeling. PLoS One 2: e1058, 2007.

38. Shen Y, Zhang J, Yu T and Qi C: Generation of PTEN knockout bone marrow mesenchymal stem cell lines by CRISPR/Cas9-mediated genome editing. Cytotechnology 70: 783-791, 2018.

39. Liu X, Chen T, Wu Y and Tang Z: Role and mechanism of PTEN in adiponectin-induced osteogenesis in human bone marrow mesenchymal stem cells. Biochem Biophys Res Commun 483: 712-717, 2017

40. Lin X, Zhou X, Liu D, Yun L, Zhang L, Chen X, Chai Q and Li L: MicroRNA-29 regulates high-glucose-induced apoptosis in human retinal pigment epithelial cells through PTEN. In Vitro Cell Dev Biol Anim 52: 419-426, 2016.

41. Sen B, Styner M, Xie Z, Case N, Rubin CT and Rubin J: Mechanical loading regulates NFATc1 and beta-catenin signaling through a GSK3beta control node. J Biol Chem 284 34607-34617, 2009.

42. Day TF, Guo X, Garrett-Beal L and Yang Y: Wnt/beta-catenin signaling in mesenchymal progenitors controls osteoblast and chondrocyte differentiation during vertebrate skeletogenesis. Dev Cell 8: 739-750, 2005.

43. Qu ZH, Zhang XL, Tang TT and Dai KR: Promotion of osteogenesis through beta-catenin signaling by desferrioxamine. Biochem Biophys Res Commun 370: 332-337, 2008.

44. Steck PA, Pershouse MA, Jasser SA, Yung WK, Lin H, Ligon AH, Langford LA, Baumgard ML, Hattier T, Davis T, et al: Identification of a candidate tumour suppressor gene, MMAC1, at chromosome 10q23.3 that is mutated in multiple advanced cancers. Nat Genet 15: 356-362, 1997

45. Whang YE, Wu X, Suzuki H, Reiter RE, Tran C, Vessella RL, Said JW, Isaacs WB and Sawyers CL: Inactivation of the tumor suppressor PTEN/MMAC1 in advanced human prostate cancer through loss of expression. Proc Natl Acad Sci USA 95: 5246-5250, 1998 .

This work is licensed under a Creative Commons Attribution-NonCommercial-NoDerivatives 4.0 International (CC BY-NC-ND 4.0) License. 